\title{
Measurements of Higgs boson properties in bosonic final states at CMS
}

\section{Filippo Errico*t}

University of Florida (US)

E-mail: filippo.erricoecern.ch

Latest results on Higgs boson property measurements in final states with photons, $\mathrm{W}$ and Z bosons are presented, using data collected by the CMS experiment in proton - proton collisions at a center of mass energy $\sqrt{s}=13 \mathrm{TeV}$ during Run 2, and combining them with data collected during Run 1 at $7-8 \mathrm{TeV}$.

European Physical Society Conference on High Energy Physics - EPS-HEP2019 -

10-17 July, 2019

Ghent, Belgium

* Speaker.

${ }^{\dagger}$ On behalf of the CMS collaboration. 


\section{Introduction}

The discovery of a new particle, compatible with the Standard Model (SM) Higgs boson, was announced in 2012 [1, 2, 3]. Since then, many efforts have been profused to study the properties of this new particle. This paper describes the latest CMS results on Higgs boson property measurements in final states with photons, $\mathrm{W}$ and $\mathrm{Z}$ bosons.

The $H \rightarrow W W$ is the second dominant decay channel with a branching fraction of $21.4 \%$ for a Higgs boson mass of $125 \mathrm{GeV}$ but the presence of the neutrino in the final state prevents the full reconstruction of the Higgs boson mass. The main (irreducible) background is the nonresonant production of $\mathrm{W}$ boson pairs, followed by the top quark contribution. Both backgrounds are evaluated from simulation and normalized from control regions in data.

The $H \rightarrow Z Z$ decay channel has large signal-to-background ratio due to the complete reconstruction of the final state decay products. The main (irreducible) background comes from the production of $\mathrm{ZZ}$ via $q \bar{q}$ annihilation or gluon fusion and it is estimated using simulation. A small contribution to the background arises from processes in which leptons are produced by heavy-flavor jets or jets misidentified as leptons: this reducible background is estimated from data.

The $H \rightarrow \gamma \gamma$ decay channel has a small branching fraction $(0.2 \%$ at $125 \mathrm{GeV})$ but profits from the clean signature and the high precision in reconstructing the diphoton invariant mass. In this case, the background is modelled from data using analytic functions.

\section{The CMS detector}

CMS (Compact Muon Solenoid) is one of the two general-purpose detectors at the LHC [4]. Its name is due to the superconducting solenoid providing an axial magnetic field of $3.8 \mathrm{~T}$ : it encloses an inner tracker, an electromagnetic calorimeter (ECAL) and a hadron calorimeter (HCAL). The muon detection system consists of up to four layers of gas-ionization chambers installed outside the solenoid and sandwiched between the layers of the steel flux return yoke. The CMS experiment uses a two-level trigger system: the first, L1, selects events of interest using information from the calorimeters and muon detectors; the second, the high level trigger (HLT), uses software algorithms accessing the full event information.

\section{Higgs boson mass}

The Higgs boson mass measurement has been obtained using the $H \rightarrow Z Z$ to four-lepton decay channel, analyzing $35.9 \mathrm{fb}^{-1}$ collected during 2016 at $13 \mathrm{TeV}$. The measurement has been performed constructing a likelihood using the mass of the four-lepton system, the mass uncertainty and a kinematic discriminant. A mass constraint on the intermediate $\mathrm{Z}$ resonance has been exploited in order to improve mass resolution. Figure 1 shows the likelihood scans as a function of four-lepton invariant mass.

The Higgs boson mass is measured to be $m_{H}=125.26 \pm 0.21[ \pm 0.20$ (stat $) \pm 0.08$ (syst) $] \mathrm{GeV}$ [5] and this is the best result up to now. 


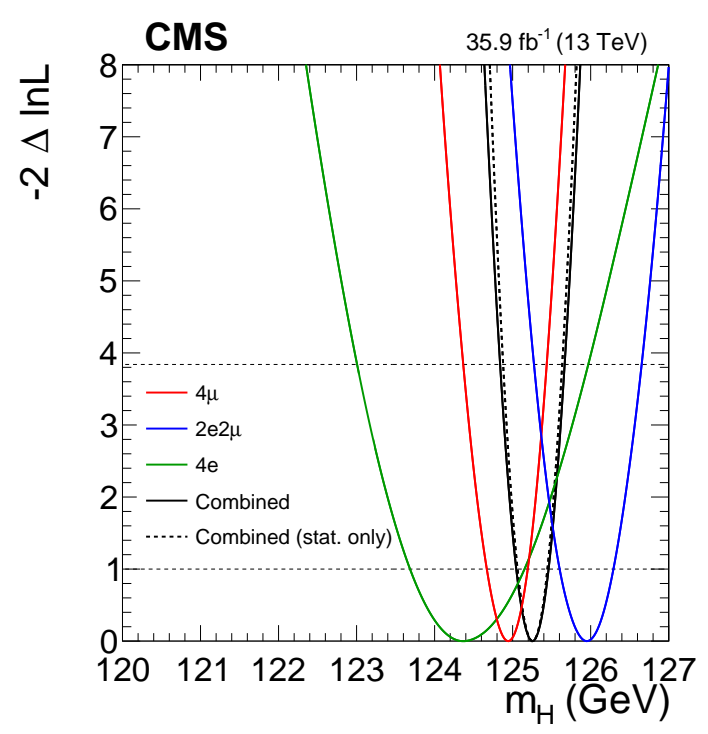

Figure 1: Likelihood scans as a function of mass for different final states and their combination. Solid lines represent scans with all uncertainties included, dashed lines instead with only statistical ones [5].

\section{Higgs boson width}

The Higgs boson width measurement suffers from the detector resolution that makes difficult a direct measurement of this property since the theoretical value of the width is $4.1 \mathrm{MeV}$ [6]. A different approach, consisting in comparing on-shell and off-shell production (4.1), has been used to extract the Higgs boson width in the $H \rightarrow Z Z$ to four-lepton decay channel, combining $77 \mathrm{fb}^{-1}$ collected during 2016-2017 at $13 \mathrm{TeV}$ with Run 1 data at 7-8 TeV. Figure 2 shows the likelihood scans as a function of $\Gamma_{H}$.

$$
\frac{\sigma_{g g \rightarrow H \rightarrow Z Z^{*}}^{\text {on-shell }} \sim \frac{g_{g g H}^{2} g_{H Z Z}^{2}}{m_{H} \Gamma_{H}}}{\sigma_{g g \rightarrow H \rightarrow Z Z^{*}}^{\text {off-shell }} \sim \frac{g_{g g H}^{2} g_{H Z Z}^{2}}{\left(2 m_{Z}\right)^{2}}}
$$

The best result up to now is $\Gamma_{H}<9.16(\exp 13.7) \mathrm{MeV}$ at the 95\% C.L. [7], setting also, for the first time, a lower bound at $0.08 \mathrm{MeV}$.

\section{Higgs boson signal strength modifier}

The signal strength modifier $\mu$ is defined as the ratio between the measured signal cross section $(\sigma)$ and its $\mathrm{SM}$ expectation $\left(\sigma_{S M}\right)$.

In the $H \rightarrow Z Z$ channel, the $\mu$ is extracted performing a multi-dimensional fit of the fourlepton invariant mass and of the kinematic discriminant $D_{b k g}^{k i n}$, using full Run 2 data, corresponding to $137.1 \mathrm{fb}^{-1}$ [8]

$$
\mu_{H Z Z}=0.94_{-0.07}^{+0.07}(\text { stat })_{-0.07}^{+0.08}(\text { syst })
$$

The signal strength modifier in the $H \rightarrow \gamma \gamma$ and $H \rightarrow W W$ decay channels has been obtained from the analysis of 2016 data. In the first, the $\mu$ has been extracted performing a binned maximum- 


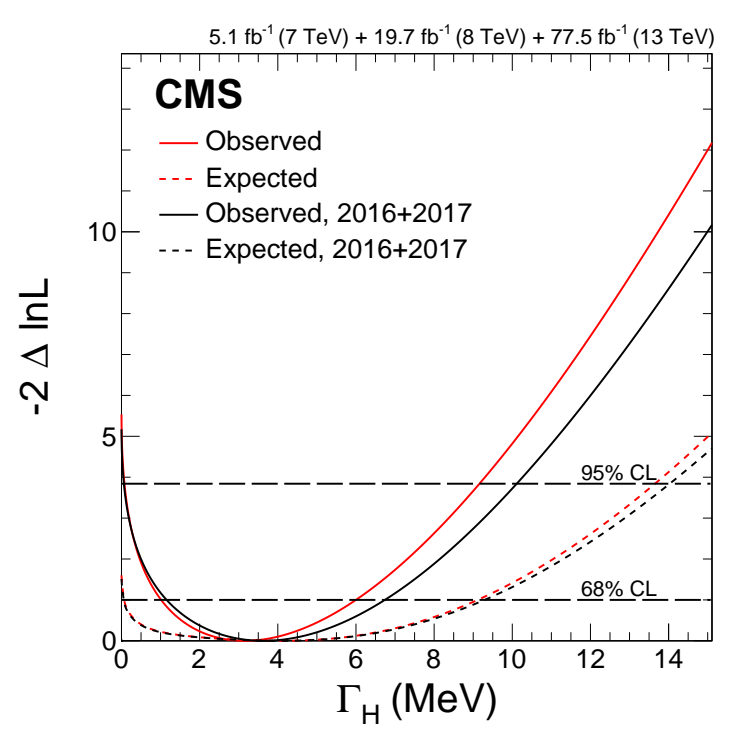

Figure 2: Observed (solid) and expected (dashed) likelihood scans as a function of $\Gamma_{H}$, using only Run 2 data (black) and their combination with Run 1 data (red) [7].

likelihood fit of the diphoton mass, with $\mu$ free to float, in the second performing a fit using simulated binned templates for signal and background processes. The results are

$\mu_{H \gamma \gamma}=1.18_{-0.11}^{+0.12}(\text { stat })_{-0.07}^{+0.09}(\text { syst })_{-0.06}^{+0.07}($ theo $), \quad \mu_{H W W}=1.28 \pm 0.10($ stat $) \pm 0.11(\text { syst })_{-0.07}^{+0.10}($ theo $)$ respectively for the diphoton [9] and for the $H \rightarrow W W$ decay channel [10]. Figure 3 shows signal strength modifiers corresponding to the main SM Higgs boson production mechanisms.

\section{Simplified template cross sections}

The Simplified Template Cross-Section approach (STXS) tries to maximise the sensitivity of the $\sigma$ measurement, minimising the dependence on the theory predictions, defining several kinematic regions using generator level information. Different stages have been developed, starting from Stage 0 , where the $\sigma$ is evaluated in regions equivalent to the different production modes, to Stage 1.1 where other regions at high $p_{T}$ or high mass have been introduced to study Beyond SM physics. Figure 4 shows the evolution of the different stages in respectively $H \rightarrow W W\left(35.9 \mathrm{fb}^{-1}\right)$ [10], $H \rightarrow \gamma \gamma\left(77.4 \mathrm{fb}^{-1}\right)$ [11] and $H \rightarrow Z Z\left(137.1 \mathrm{fb}^{-1}\right)$ [8] processes. Due to large statistical uncertainties, some bins were merged in the fit.

\section{Fiducial cross section}

The fiducial cross section is a cross section in a defined fiducial phase space. This volume is set by criteria at generator level based on kinematic, geometrical variables and on the topology of the event, in order to minimise the dependence on theoretical uncertainties. In the $H \rightarrow Z Z$, the fiducial volume is defined in a unique way for all the observables. In the $H \rightarrow \gamma \gamma$, the phase space is 

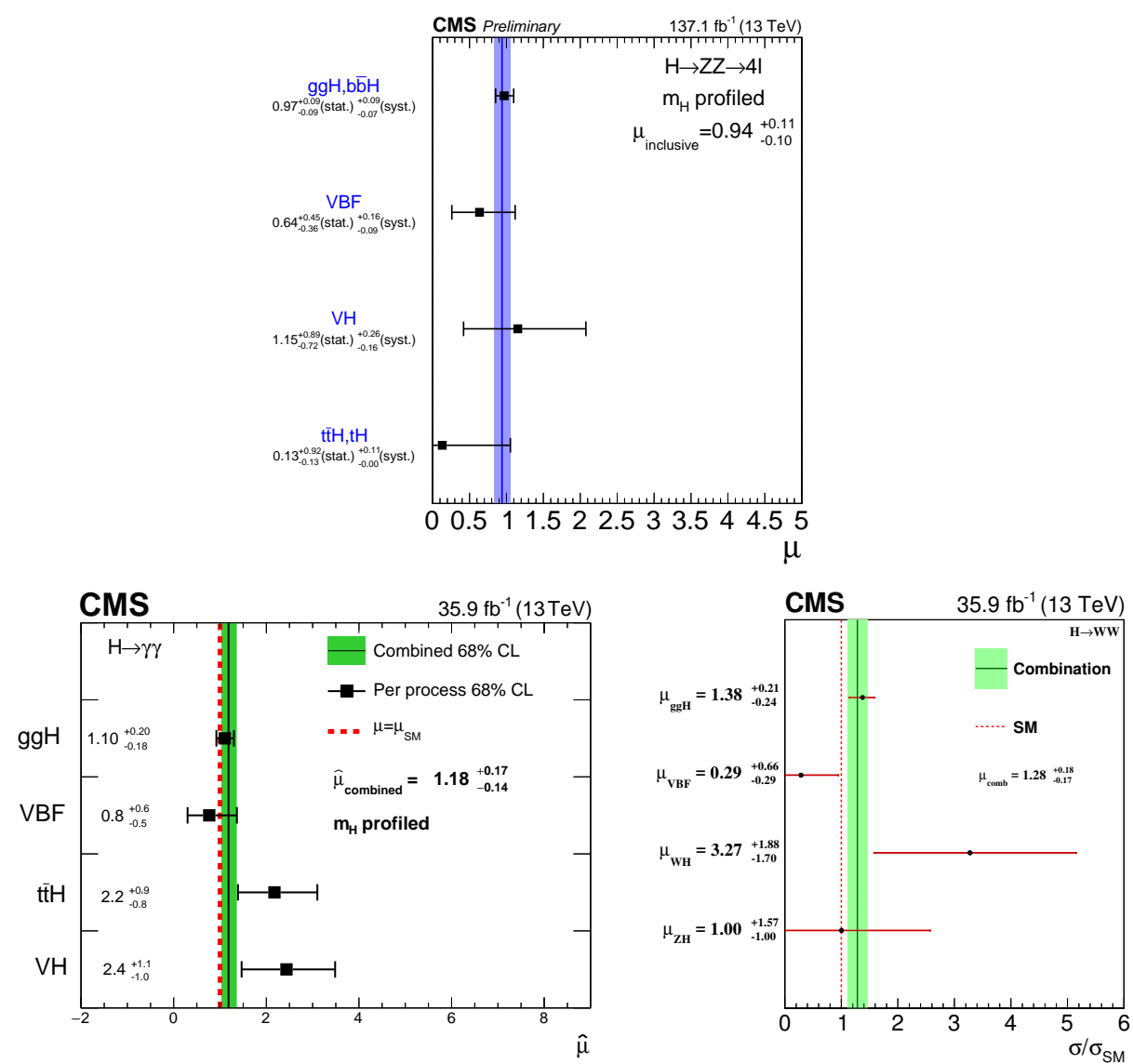

Figure 3: Observed signal strength modifiers corresponding to the main SM Higgs boson production mechanisms: (top) four-lepton decay channel [8], (bottom left) $H \rightarrow \gamma \gamma$ [9], (bottom right) $H \rightarrow W W$ [10]. The vertical line stands for the combination; the horizontal bars and filled band indicate the $\pm 1 \sigma$ uncertainties, including both statistical and systematic sources.

tuned according to the observable under study. Figure 5 shows the inclusive fiducial cross section for both four-lepton $\left(137.1 \mathrm{fb}^{-1}\right)[8]$ and diphoton $\left(35.9 \mathrm{fb}^{-1}\right)$ [12] final states. In both channels, the measurement is found in agreement within the uncertainties.

\section{Summary}

Measurement of the Higgs boson properties in the bosonic final states at CMS have been presented. The Higgs boson mass and width measurements are the best results up to now, respectively $m_{H}=125.26 \pm 0.21 \mathrm{GeV}$ and $\Gamma_{H}<9.16 \mathrm{MeV} @ 95 \%$ C.L. Latest CMS results on the signal strength modifier, on the STXS and on the fiducial cross section have been also shown: no deviations from SM expectations have been observed. 

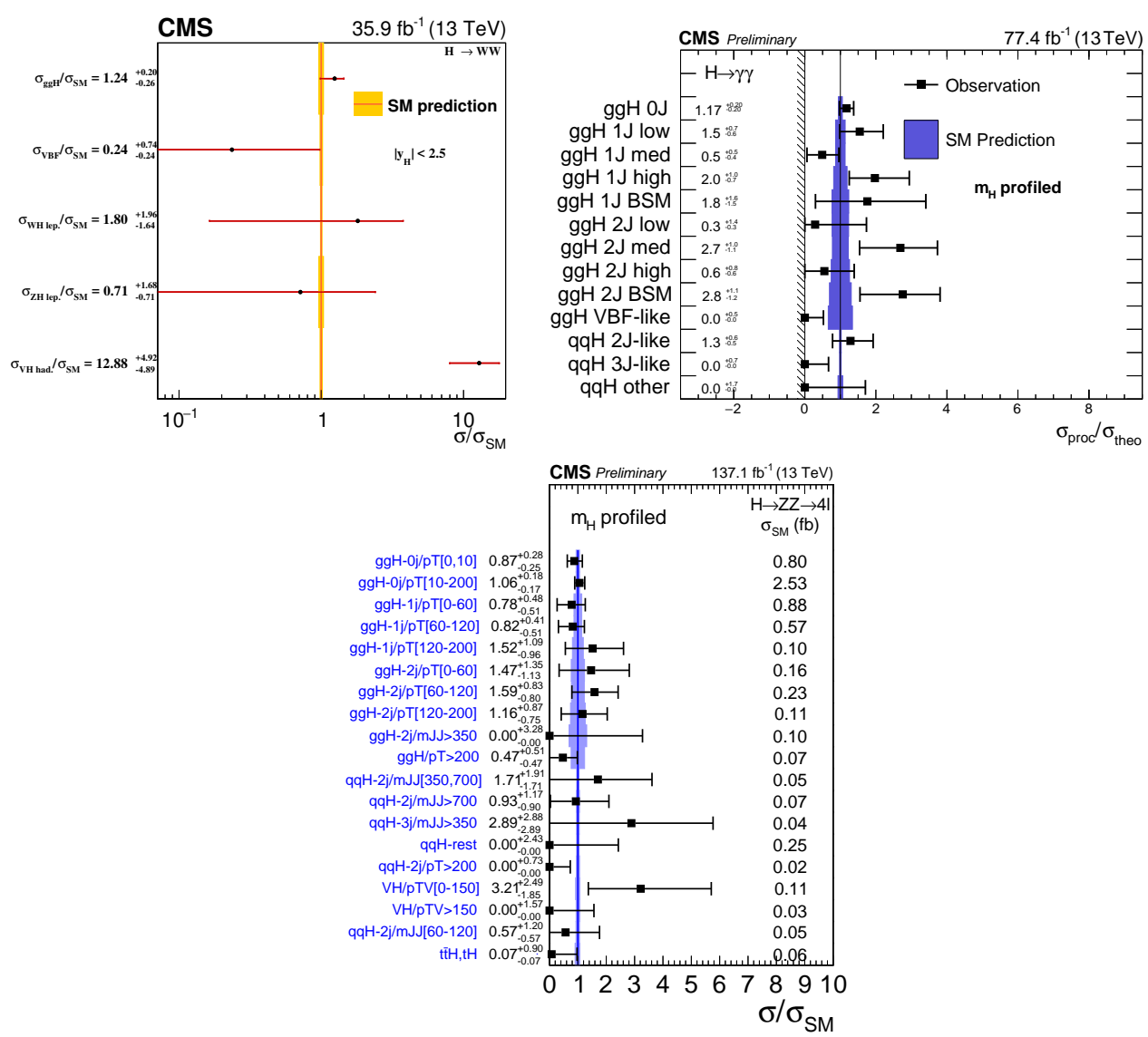

Figure 4: The ratio between $\sigma$ and $\sigma_{S M}$ for different stages in the STXS: (top left) $H \rightarrow W W$ Stage 0 [10], (top right) $H \rightarrow \gamma \gamma$ Stage 1 [11] and (bottom) $H \rightarrow$ ZZ Stage 1.1 [8].
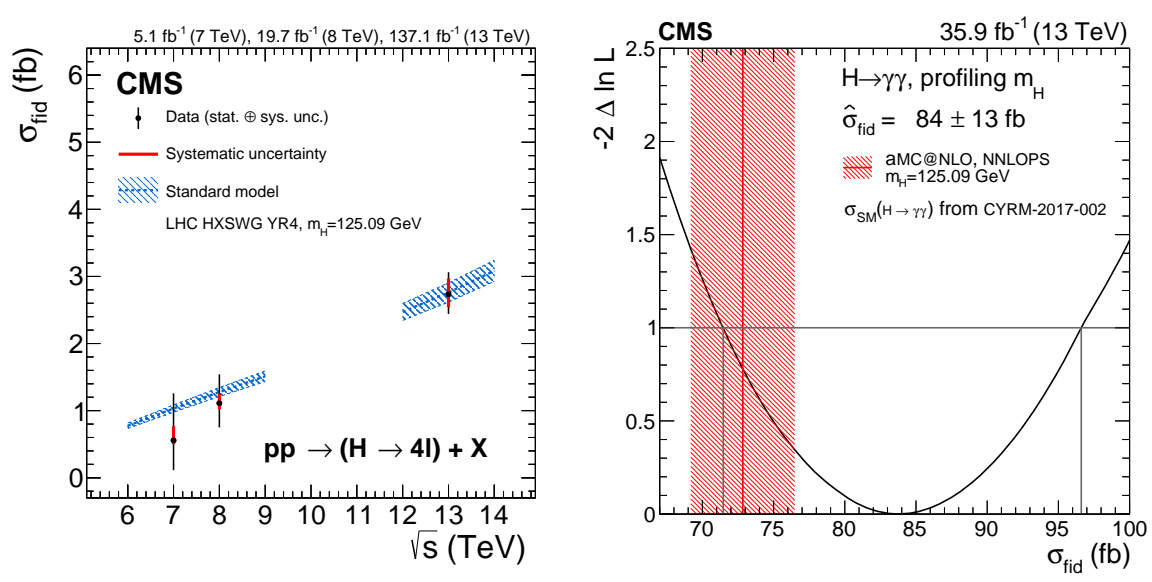

Figure 5: (Left) Inclusive fiducial cross section as a function of $\sqrt{s}$ in the four-lepton final state [8]. (Right) Likelihood scan for the fiducial cross section in the diphoton channel [12]. 


\section{References}

[1] CMS Collaboration, "Observation of a new boson at a mass of $125 \mathrm{GeV}$ with the CMS experiment at the LHC", Physics Letters B716 (2012)

[2] CMS Collaboration, "Observation of a new boson with mass near $125 \mathrm{GeV}$ in pp collisions at $\sqrt{s}=7$ and 8 TeV", JHEP 06 (2013) 081

[3] ATLAS Collaboration, "Observation of a new particle in the search for the Standard Model Higgs boson with the ATLAS detector at the LHC", Phys.Lett. B716 (2012)

[4] CMS Collaboration, "The CMS experiment at the CERN LHC", JINST 3:S08004,2008.

[5] CMS Collaboration, "Measurements of properties of the Higgs boson decaying into the four-lepton final state in pp collisions at $\sqrt{s}=13 \mathrm{TeV} "$, JHEP 11 (2017) 047.

[6] C. Patrignani et al. "Particle Data Group", Chin. Phys. C, 40, 100001 (2016) and 2017 update.

[7] CMS Collaboration, "Measurements of the Higgs boson width and anomalous HVV couplings from on-shell and off-shell production in the four-lepton final state", Phys. Rev. D 99, 112003 (2019).

[8] CMS Collaboration, "Measurements of properties of the Higgs boson in the four-lepton final state in proton-proton collisions at $\sqrt{s}=13 \mathrm{TeV}$ ", CMS-PAS-HIG-19-001, https://cds.cern.ch/record/2668684

[9] CMS Collaboration, "Measurements of Higgs boson properties in the diphoton decay channel in proton-proton collisions at $\sqrt{s}=13 \mathrm{TeV}$ ", JHEP 11 (2018) 185

[10] CMS Collaboration, "Measurements of properties of the Higgs boson decaying to a W boson pair in pp collisions at $\sqrt{s}=13 \mathrm{TeV}$ ", Phys. Lett. B 791 (2019) 96

[11] CMS Collaboration, "Measurements of Higgs boson production via gluon fusion and vector boson fusion in the diphoton decay channel at $\sqrt{s}=13 \mathrm{TeV}$ ", CMS-PAS-HIG-18-029, https://cds.cern.ch/record/2667225

[12] CMS Collaboration, "Measurement of inclusive and differential Higgs boson production cross sections in the diphoton decay channel in proton-proton collisions at $\sqrt{s}=13 \mathrm{TeV}$ ", JHEP 01 (2019) 183. 